\title{
Responding to mental health vulnerability in Maltese detention centres: the use of psychological support groups as an intervention tool
}

\author{
Julian Caruana ${ }^{1,2^{*}}$ (1) and Alexia Rossi ${ }^{1,2}$
}

\begin{abstract}
Maltese immigration law means that individuals seeking asylum in this country undergo a period of mandatory detention upon arrival lasting a maximum of 18 months. During their stay in detention centres, these individuals are exposed to an environment characterised by loss of liberty, prolonged inactivity, disconnection to family and the outside world and lack of adequate information about ongoing legal proceedings. It is hardly surprising that such a prolonged period of limbo, awash with isolation, uncertainty and degradation, has a negative impact on the mental health of individuals who, in the vast majority, have already experienced multiple personal losses. Research has in fact indicated that the majority of migrants detained in Europe reported deterioration in their mental health during their stay in detention. This article explores the impact of psychological support groups provided by nonstate actors in Maltese detention centres aimed at educating about mental health issues, as well as at providing participants with a space where they can openly discuss their psychological difficulties and collaboratively explore ways to enhance their mental health within the constraints of detention. This paper discusses the challenges and benefits of conducting such a programme with an emphasis on how participants viewed the opportunity provided. This discussion analyses how these groups functioned as a medium enabling participants to voice their phenomenological experience of detention on both an individual and collective level and as an arena for negotiating a sense of self that is resilient to the experience of forced detention. In conclusion, this paper provides suggestions on how to address the psychological needs of migrants in closed centres.
\end{abstract}

Keywords: Detention, Asylum seekers, Mental health, Group intervention

\section{Introduction}

\section{Overview of migration flows into Malta}

Every year, scores of migrants reach the Maltese islands, approximately 15,000 in the decade between 2002 and 2012 (Human Rights Watch 2012). Until 2002, the number of asylum seekers ${ }^{1}$ arriving in Malta was relatively small, but a sudden change in migration patterns in the Mediterranean saw a sharp increase in the amount of migrants arriving in Malta irregularly by boat from North Africa. Since 2002, the vast majority of migrants reaching Malta's shores arrived by boat from Libya. ${ }^{2}$ Annual arrivals have since totalled an average of 1500-1700

\footnotetext{
* Correspondence: julian.j.caruana@um.edu.mt

'Jesuit Refugee Service Malta, SAC Complex, Triq ix-Xorrox, B'Kara, Malta

2University of Malta, Msida, Malta
}

individuals, with the exception of 2010 when the implementation of Italy's pushback policy reduced the arrival numbers substantially. Most hail from Sub-Saharan Africa with the largest national groups being Somali (32\%) and Eritrean (14\%), followed by Nigerian (6\%) and Sudanese $(5.3 \%)^{3}$ Adult males account for the majority of these arrivals, but a significant number of women and accompanied/unaccompanied minors are also represented. ${ }^{4}$ Often described as "mixed flows" due to their different motivations for heading to Europe, many of these undocumented migrants have suffered countless traumas in their country of origin or other transit countries, including war, torture and serious human rights violations. More specifically, a number of women are victims of sexual violence, abuse and trafficking. 
Almost all of these migrants apply for asylum upon arrival thus setting in motion a number of processes dictated by Maltese immigration law that will ultimately determine who will be granted international protection in Malta. Since 2002, around 56\% of arrivals have been granted some form of protection (Jesuit Refugee Service Malta 2013).

\section{Maltese immigration law and administrative detention}

Maltese immigration law dictates that every individual who enters or is present in Malta without authorisation is subject to a removal order. The majority of asylum seekers are in fact unauthorised boat arrivals (85\% of asylum applicants in 2011) to whom this removal order is issued. Once ordered, mandatory detention is then triggered until this person is removed from Malta as enshrined by Article 14(2) of Malta's 1970 immigration act. Alongside this, all third country nationals may apply for international protection in Malta. Once an individual applies for asylum, their duration in detention will be determined by the time taken to process their claim and the outcome of their application. The two forms of international protection granted by Maltese law are refugee status $^{5}$ and subsidiary protection. ${ }^{6}$ The Maltese asylum procedure also allows for the granting of Temporary Humanitarian Protection (THP) to those who do not qualify for international protection, but cannot be returned to their country because of reasons related to age, physical and mental health or other humanitarian considerations (Jesuit Refugee Service Malta 2008). Should a person's claim for asylum be rejected, the individual has the right to submit an appeal to the refugee appeal's board.

Should the individual's asylum claim also be rejected at appeal, immigration authorities may proceed with repatriation. However, the maximum detention allowed by law is 18 months, and if repatriation has not been effected during this time, these individuals are released to live in the community. The only exception to detention that exists is for those individuals, who after an assessment of their situation, are deemed to be vulnerable due to age, disability, pregnancy or chronic/serious physical and/or mental health problems, a process which itself can take up to 6 months. In accordance with government policy, vulnerable individuals are not detained and are hence released even if their asylum application is still pending.

In Malta, migrants and asylum seekers are detained in one of two facilities. Both are military compounds in the south of the island, which together with fulfilling their military function also contain specifically designated areas for the detention of migrants. These include warehouses and smaller secure units, each equipped with rudimentary facilities including dormitory-style quarters for sleeping, a room furnished with tables and a television for indoor recreation, toilets and showers and a basic kitchen area. In one of the facilities, the areas of detention are each annexed to a small outside area to which migrants have constant access during the day. In the other facility, detained individuals are given an hour of access daily to a small secure outside area for recreation purposes. Both facilities are staffed exclusively by ex-security personnel. Almost all are male, with no specific training in the care of immigrants or vulnerable individuals. Whilst single men, women and couples are detained in different units, differentiation is not usually made in terms of nationality, length of time in detention, status of asylum application, and hence, individuals with different religions, customs, backgrounds and prospects for protection generally find themselves sharing the same confined space for months on end. Detained migrants are provided only the most basic of servicesshelter, food and health care-and there is no existing structure for the provision of recreational and educational activities.

Malta's administrative detention policy and practice has been strongly attacked in recent years by local NGOs, as well the international community through monitoring agencies such as the Council of Europe's Committee for the Prevention of Torture. Human rights organisations have highlighted the conditions in Maltese detention centres as particularly poor and slated the lack of privacy, substandard sanitary conditions and insufficient access to recreational and educational activities (Amnesty International 2013). Moreover, European Court of Human Rights' judgements have repeatedly harshly criticised and questioned the detention system (Jesuit Refugee Service Malta 2014).

\section{Mental health challenges faced by asylum seekers}

It is well understood that becoming a refugee is a composite experience (Watters 2007) encompassing numerous losses and hardships that occur across the pre-migration, flight and post-migration periods. The decision to flee in itself brings about the loss of homeland, culture, tradition and a familiar way of life. Individuals seeking refuge may have also been exposed to several traumatic experiences in their country of origin such as the loss of family due to war or oppression, being recruited as a soldier to fight or having to live in hiding without the chance to exercise one's rights. Furthermore, they may have endured traumatic experiences as they fled their country such as imprisonment, torture, rape, lengthy and arduous journeys and witnessing the death of friends or family en route. Subsequently, the post-migration environment is itself fraught with a wide array of adversities including stringent asylum policies, detention practices and challenges such as the navigation 
of an alien cultural context (Silove et al. 2000; Castro and Murray 2010).

These prolonged and acutely distressing events, in particular the myriad losses of home, status and culture synonymous with this experience, have been associated with powerful demands on the individual's psychological systems; examples offered include the upheaval of one's identity (Alcock 2003) and the loss of meaning and hope (Fischman 2008). Empirical evidence has highlighted the significant impact of refugee experiences on mental health, uncovering an association between these experiences and feelings of powerlessness (Farias 1991, as cited in Muecke 1992), uncertainty, dependency and of being in the minority (Hussain and Bhushan 2009). In fact, a meta-analysis of 181 surveys investigating the mental health of 81,866 refugees (Steel et al. 2009) found high prevalence rates of post-traumatic stress disorder (PTSD) (30.6\%) and depression (30.8\%) in this population. On the other hand, other systematic meta-analyses of the mental health of refugees (Fazel et al. 2005; Bogic et al. 2015) pointed towards lower prevalence rates. These meta-analyses indicated that major depression rates in refugees are similar to those in the general population in Western countries and that the prevalence rate of PTSD in this population stands at 9\%. The latter rate however still means that refugees are about ten times more likely than the age-matched general population to suffer from PTSD. It is also relevant to note another meta-analysis uncovered significantly worse mental health outcomes for refugees when compared against a group of non-refugees (e.g. voluntary migrants) (Porter and Haslam 2005).

Available evidence seems to indicate that, at the very least, asylum seekers are significantly more at risk of suffering from post-traumatic stress than the general population or the migrant population at large. Furthermore, it is important to point out that, whilst there is no available evidence of prevalence rates of mental health disorders among detained asylum seekers, it is probably fair to assume that the experience of captivity and deprivation of liberty, within the process of seeking protection, would most probably act as an added stressor.

\section{The psychological impact of detention}

A ten-study meta-analysis specifically investigating the impact of immigration detention on asylum seekers indicated an association between this practice and poor mental health outcomes, with the presence of high levels of anxiety, depression and PTSD being observed in all studies (Robjant et al. 2009).

Detention has been described as a "system that by its very nature causes psychological harm" (Fazel and Silove 2006, p. 252) and considerable literature has shown that it may have an adverse effect on a refugee's mental health (e.g. Silove et al. 2000) either through the stressors it imparts as an institution or by compounding the trauma experienced by individuals prior to arrival. Whilst in detention, migrants are commonly exposed to an environment characterised by loss of liberty, disconnection to family and the outside world, harsh treatment or abuse from staff, prolonged inactivity and lack of adequate information or knowledge about one's legal situation (Jesuit Refugee Service Europe 2010a; Fazel and Silove 2006; Keller et al. 2003). This often results in an atmosphere of mistrust, uncertainty and arbitrariness for the individual where they may feel degraded, undignified and isolated.

Such environments, aptly referred to by Goffman (1961) as "total institutions" (p.68) have an uncanny ability to strip an individual of that which allows the maintenance of a healthy sense of self; choices, status, support structures and freedom by exposing him/her to the prolonged cessation of past roles, loss of individuality, connection to the outside world and extreme loss of control over their safety, presentation and life path. The state that the migrant is hence forced into can have a profound effect on his/her sense of self, triggering the emergence of associated psychological issues.

In this context, the potential deleterious impact of detention on the individual can be more effectively understood and one is left with little question as to why observations of detainees have indicated experiences of despair, profound hopelessness and suicidal ideation and why mental health problems such as depression, panic disorder and PTSD are increasingly prevalent (Salinsky and Dell 2001; Pourgourides et al. 1996).

Furthermore, the fact in itself that the asylum procedure requires the individual to be subjected to long-term mandatory detention, can have adverse longstanding effects on the asylum seeker's well-being. Research on Iraqi asylum seekers in the Netherlands has indicated that a prolonged and stressful asylum procedure tended to have a long-term deleterious impact on the individual's mental health and quality of life in general. Laban, Gernaat, Komproe and van der Tweel in 2006 showed that asylum procedure stress was among the factors that had the highest odds ratios for psychopathology in this population. Furthermore, Laban, Komproe, Gernaat and de Jong (2008) reported significantly lower quality of life $(\mathrm{QoL})$, higher functional disability and more physical complaints among respondents with a long asylum procedure, with length of stay being the strongest predictor for a low overall QoL.

\section{Maltese detention centres: mental health concerns}

An in-depth review of administrative detention practices in Malta (Jesuit Refugee Service Europe 2010b) highlighting the perspective of 89 detained migrants 
brought to the fore the deleterious impact of several factors. The physical conditions in the Maltese centres were viewed as highly distressing with around $75 \%$ of interviewed migrants speaking of overcrowding, poor sanitation, restricted space and lack of privacy, with these conditions being associated with outcomes like deprived sleep and increased incidence of infection. Access to basic services and activities was described as being poor or non-existent, with $50 \%$ citing access to medical staff once a week and $25 \%$ less than once a month. Eighty percent reported having no access to activities such as education, only 50\% reported free access to outdoor space and none reported access to internet or a computer. For most, even the most basic sources of support were denied; $80 \%$ stated that they were unable to keep in touch with their family due to scant access to the phone, limited phone credit provided or the confiscation of contact numbers with their general belongings upon arrival. Moreover, only $4 \%$ reported receiving visits from family members as most centres do not allow visits of a personal nature.

The social conditions also appeared to add to the suffering these detained migrants experienced, with almost half of the respondents drawing attention to the arbitrary and inadequate application of rules. Across detention centres, there was generally a low staff- detainee ratio with the former being generally ill-equipped, reticent and powerless to change the situation to meet the latter's needs. These social conditions generally led to an atmosphere where detainees felt unsafe and undignified, with $28 \%$ reporting physical assault, mostly by other detainees, and a considerable number reporting verbal abuse from detention staff. This sense of insecurity was further exacerbated by the procedures governing the detention of migrants leading to the relinquishing of any sense of control they have over their situation. More than half of those interviewed expressed being unaware of the reason for their detention, almost $80 \%$ were distressed by the scant information on procedures and the duration of their detention and only $14 \%$ were aware of their release date.

The results of this comprehensive research strongly indicate that the physical and social conditions in Maltese detention centres appeared to have a tangible effect on migrants, with $62 \%$ reporting the emergence of physical health problems and $80 \%$ reporting deterioration in their mental health since their arrival. The general perception is that the poor living conditions, separation from the outside world and loved ones, incarceration and worry related to asylum procedures concomitant with the Maltese detention system (Jesuit Refugee Service Europe $2010 \mathrm{~b})$ are at the root of a discernible deterioration in the psychological well-being of asylum seekers in Malta.

\section{Group interventions for refugees and asylum seekers}

Whilst research on group therapy with asylum seekers and refugees suffering from mental health difficulties is scarce, it has been suggested that group interventions focusing on empowerment (Curling 2005) and support (Manneschmidt and Griese 2009) may be effective.

One of the few group interventions that is described and analysed in depth by empirical literature is a multifamily education and support group conducted with Bosnian refugee families in Chicago, USA, across several years. Weine et al. (2004) conducted a qualitative analysis of the field notes from these group meetings and concluded that several points of potential psychological difficulty, could be effectively addressed through family interventions such as helping parents and children share memories in ways that are developmentally timed and foster trust. Furthermore, a quantitative analysis carried out by Weine et al. (2008) of the same kind of group intervention with Bosnian adult refugees with PTSD and their families, indicated that a multiple-family group was effective in increasing access to mental health services.

In another study, Droždek et al. (2012) provided evidence that a group intervention combining psychodynamic, cognitive-behavioural, and supportive approaches, in conjunction with nonverbal treatments, led to a significant improvement in the mental health status of Iranian and Afghani asylum seekers and refugees diagnosed with PTSD in the treatment group when compared to the control group.

\section{Statement of purpose}

In light of the potential deleterious psychological effects of administrative detention on asylum seekers arriving in Malta, this article aims to outline and evaluate the implementation of psychological support groups as an intervention tool to address mental health vulnerability in this context.

\section{Background to psychological support groups Jesuit Refugee Service, Malta}

The Jesuit Refugee Service (JRS) is an international organisation that seeks to accompany, serve and advocate on behalf of refugees and forcibly displaced persons. JRS Malta strives to provide legal and psychosocial assistance to asylum seekers who arrive on this island with a particular emphasis on addressing the needs of those detained in closed centres. Close contact with individuals detained for months in these centres has made JRS acutely aware of the psychological difficulties these persons face and the myriad adverse mental health consequences they suffer from. In response to this need, over the recent years JRS Malta has strived to offer professional psychological services to migrants in detention and one of the initiatives implemented in this regard was the organisation of psychological support groups. 


\section{Aim of psychological support groups}

The rationale for considering some form of group intervention was that such a format could constitute an efficacious way of offering professional psychological assistance to a wide range of individuals and that engaging on a collective level could be advantageous in terms of activating/augmenting mechanisms for reciprocal support and offering the opportunity for vicarious learning. Moreover, group work has been described as an appropriate modality for working with refugees due to those characteristics it shares with the collectivist cultures many of these refugees hail from (Lim and Koike 2010). More specifically, psychological support groups were considered as a useful tool for reaching the following explicit aims: Firstly, educating about mental health issues and challenging stereotypes of persons experiencing mental health problems. Secondly, providing participants with a space where they can openly express themselves and discuss their experience of detention and psychological difficulties. Lastly, to impart a set of coping skills and strategies that can enhance the detainee's capacity to take care of his/her mental health and additionally to provide a framework through which detainees can collaboratively explore ways to enhance their mental health within the constraints of detention.

\section{Method}

\section{Programme structure}

The group format adopted was tailored to reach the aforementioned aims and was composed of three main components (refer to Table 1):

1. A psychoeducative aspect comprising learning about mental health difficulties and disorders, psychiatric and psychological treatment and recovery from mental health problems. This component aimed to tackle misconceptions that fuel stereotypes by offering the opportunity for participants to reflect on and discuss their personal understanding of mental health and illness.

2. A component dedicated to imparting behavioural and cognitive coping strategies with an emphasis on discussing, testing and reviewing strategies applicable in a closed detention centre. This aspect adopted an explicit strengths-based focus and aimed to encourage attendees to share their suggestions for coping.

3. A phenomenological element aimed at creating the appropriate space for participants to give voice to their experience of being detained and to speak about any aspects of their reality they wished to share.

The programme's learning content was mainly based on cognitive-behaviour principles and various techniques were used to reach the stated aims including large and small group discussions, role plays and experiential exercises. A cognitive-behaviour theoretical background was considered beneficial in this case due to available empirical evidence confirming this modality's effectiveness when working with a refugee population. For example, Otto et al. (2003) concluded that cognitive behaviour therapy (CBT) has been used with a certain degree of success as a therapeutic mode with refugees exposed to traumatic experiences. Furthermore, a strong evidence base exists for group CBT's efficacy in treating anxiety disorders (e.g. White et al. 1992; Main et al. 2005) and depression (e.g. Huntley et al. 2012). Hence, the programme was structured into a set of sessions covering a range of psychological topics with an underlying cognitive-behavioural focus, with each session comprising elements from the three components.

\section{Implementation}

Nine psychological support groups composed of an average of 15 weekly sessions of a 1-h duration were implemented over a 2-year period starting from 2012. These support groups were open to all individuals residing in Maltese detention centres. Two counselling psychologists were recruited to fulfil the role of facilitators, and arrangements were made to ensure the availability of cultural mediators. ${ }^{7}$ All programmes began with an information session in a detention centre, delivered through the necessary cultural mediators, where the aim of these groups together with an overview of the content was provided, following which interested individuals were invited to complete an interest form. Groups were subsequently set up based on the number of participants interested and their language preferences, with the average group size being 10-15 individuals. Language

Table 1 Topics within the psychological support group

\begin{tabular}{llll}
\hline Psychoeducation & Cognitive-behavioural skills & Phenomenological processing & Interpersonal skills \\
\hline Introduction to mental health & Positive thinking & Experience of detention & Communication \\
Depression and anxiety & Emotional management & Suffering and hope & Group dynamics and social skills \\
Post-traumatic stress & Coping strategies & Meaning in life & \\
Mental health stigma & Goal setting & & \\
\hline
\end{tabular}


preferences were French, Amharic, Tigrinya and Somali, and interpretation in these languages was catered for.

The location of the group sessions varied depending on the centre. In some cases, the availability of a classroom external to individuals' living quarters allowed facilitators to conduct the session there. In other cases, the sessions were conducted in the recreation area within the living quarters. Given that a large proportion of the detention population were non-English speakers, participants were often grouped by language preference and a cultural mediator was present to provide interpretation in the chosen language.

\section{Evaluation method}

Given that this was the first programme of its kind organised in Maltese detention centres, evaluating the psychological support groups' impact was considered of paramount importance. The rationale for evaluating this intervention was to gather evidence to guide future decision making about whether this programme should be repeated as an intervention and, if so, whether any amendments are required. Having said this, when considering how to evaluate the impact of this intervention on participants, given the context that beneficiaries were a generally distressed, diffident and to some extent disoriented detainee population, importance was given to choosing an evaluation method that minimised the level of intrusiveness. It was therefore deemed appropriate to implement a mainly qualitative form of evaluation that focuses on personal experience and self-reported effects. In this approach, evaluating impact was framed as understanding participants' phenomenological experience of the sessions and their learning about mental health and their own role in taking care of their psychological well-being.

To this end, a tailor-made participant feedback form containing a set of scales and open-ended questions was deemed the most suitable means for gathering pertinent evidence. The first section of the feedback form comprised questions regarding basic demographic information and session attendance. The subsequent section contained questions about the perceived utility of the sessions and materials provided answerable on a scale from 0 to 10; 0 representing "not helpful at all" and 10 representing "very helpful". The last part of the form contained a series of open-ended questions about participants' perceived learning outcomes, preference of sessions and suggestions for programme improvement.

This form was administered to every group during the last session of the programme with cultural mediators translating the questions to non-English speakers at the onset and also being available to assist whoever asked for further support. Participants had the option of writing their answers in their native language and these responses were then translated to English by the cultural mediators.

\section{Results}

A total of 87 participants completed the feedback form with more than half of these respondents having attended six or more sessions of the programme. The majority of respondents were male and below 30 years of age (refer to Table 2), mirroring both the composition of participants in the psychological support groups conducted and the general detention population.

Regarding the take-up of the programme, 190 interest forms were completed initially, 174 individuals attended the first session and 87 attended the last session. The average attendance per session across the 9 groups was of 13 participants and the mean attendance for the whole programme was of 113 asylum seekers. Comparing this mean, with the average immigration detention population during the implementation period of 900 persons, indicates that approximately $13 \%$ of the total detention population regularly attended the group sessions.

The mean score of participants' ratings of the utility of sessions conducted $(\mathrm{M}=9.16, \mathrm{SD}=0.24)$ and materials provided $(\mathrm{M}=9.13, \mathrm{SD}=0.19)$ both point towards a highly favourable view of the programme's value. The most common responses regarding session preference concerned the topic of mental health problems and

Table 2 Demographic information

\begin{tabular}{lll}
\hline Characteristic & Frequency & Percentage \\
\hline Gender & 76 & 87.4 \\
Male & 11 & 12.6 \\
Female & 87 & 100.0 \\
& & \\
Age & 74 & 85.1 \\
16-30 & 8 & 9.2 \\
$31-45$ & 0 & 0.0 \\
$46-60$ & 0 & 0.0 \\
$61+$ & 5 & 5.7 \\
NA & 87 & 100.0 \\
& & \\
Mean (23.56) & & \\
Range (16-40) & & 65.5 \\
Country of origin by region & & 26.4 \\
East Africa & 57 & 1.1 \\
West Africa & 23 & 6.9 \\
Asian & 1 & 100.0 \\
NA & 6 & \\
& 87 & \\
\hline
\end{tabular}


stigma followed by the sessions on positive thinking and expressing emotions. The most frequently mentioned outcomes concerned acquiring general knowledge about psychology/mental health, learning how to enhance interpersonal relating and engage in positive thinking, and achieving better emotional management. Finally, in response to the question "Would you change anything about this programme", 39 participants wrote that they would keep the programme as is and 26 either suggested that the number of sessions should be increased or that the session time should be increased.

A number of themes characterising the phenomenological experience of detention emerged clearly during the sessions. Naturally lack of freedom was one of the most commonly mentioned themes; participants spoke about losing the ability to choose how to use their time and losing the faculty to attempt to shape one's life. In this regard, freedom emerged as a fundamental element of being human, with detainees expressing the idea of experiencing an impoverished version of living. Participants also spoke about past losses being aggravated by the experience of being detained. They spoke of a loss of time that will never be recuperated being added to their long list of personal losses, with the experience of detention thus intensifying their sense of loss.

Being treated as a criminal was another prevalent theme with attendees repeatedly employing phrases such as; "This is the first time I am in prison" or "I never thought that I will end up in prison". In certain cases, the perception of being regarded as a criminal seemed to be particularly distressing for the individual as it acted as a challenge to their previous self- concept. Feeling less of a human was another prevalent topic in participants' discourse. Participants often mentioned feeling that they are not afforded the respect they deserve as humans and that the life they are leading is unfit for a human being. The poor living conditions (even the architecture of the detention centre itself with some zones being originally constructed to function as a warehouse) and the collective and impersonal manner detainees were treated in contributed to this feeling. For example, incidents such as solely being referred to by number were often described as inhuman and degrading.

Uncertainty about the future was another frequently cited topic. More specifically, attendees expressed uncertainty about the outcome of their asylum application and the associated fear of being deported back to one's country of origin. This uncertainty seemed to create a climate of insecurity in detention; a general feeling of being in transition, of not having reached safety despite surviving the boat journey and arriving in Europe.

On a more symptomatic level a number of psychological difficulties were commonly expressed: depressed mood, insomnia, lethargy, anxiety and its physical symptoms and post-traumatic stress symptoms including intrusive recollections of past events, recurrent nightmares and hypervigilance to threat. These difficulties appeared to be to some extent related to the phenomenological experience of detention as described above, with participants speaking about viewing detention either as cause or an exacerbating factor.

\section{Discussion}

The psychological support groups provided insight into the lived experience of detention and the various psychological difficulties migrants detained in closed centres face. This section will seek to explicate on these issues, evaluate the impact of this intervention tool on participants, as well as discuss the overall benefits and challenges of conducting such a programme.

\section{Psychological support groups as an intervention tool: benefits}

The psychological support groups appeared to function as a useful tool for providing mental health care to migrants detained in closed centres. Interestingly, participants spoke about seeing these groups as being advantageous both for their life in detention and for their life in general; "I learnt how to cope with detention"; "I learn how to manage myself in detention"; "How to solve my life with programme, to have goals, to help myself with psychology". Participant feedback seems to indicate that the programme had a salutary psychological impact by making attendees more aware and knowledgeable about the concept of mental health and related issues and by augmenting their ability to take care of their psychological health through the acquisition of relevant cognitive and behavioural strategies. In this regard, feedback highlighted the development of strategies facilitating emotional management, balanced thinking and interpersonal relating. Declared session preference appears to reinforce the hypothesis that participants found particularly valuable the aspects of the programme addressing these areas.

Participants spoke about the various ways the programme helped them develop greater emotional intelligence. They mentioned becoming better able to foster positive emotions; "I found it useful to learn patience"; "I learnt a lot of things, how I can keep in high spirits", to deal with negative emotional experiences; "How to get out of depression by sharing your problems with people" and showed an overall greater confidence in managing emotions; "I learnt how you can rest your mind". More specifically, the programme's capacity to facilitate the fostering and/or maintenance of hope emerged as a particularly strong benefit; "It helped me because it gives me hope."; "That in life you may lose the people you have, you may lose the things you have, no 
matter what happens never lose yourself/hope." The programme also appeared to contribute to changes in the cognitive domain. Following completion of the programme, detainees spoke of being more knowledgeable of the relationship between cognitions and emotions and being able to access alternative interpretations of current situations; "To know how to steer your life, if you have reject to know that time come to have freedom".

Another benefit emphasised by participant feedback concerned progress in the interpersonal dimension. The group sessions seemed to have provided participants with a fruitful opportunity to enhance their interpersonal skills; "I learnt how deal with the people" "How to approach our neighbour" "How to live with future people I meet", and at the end of the programme attendees reported greater confidence in their ability to engage productively on a collective level "I know a lot about... how to solve problems together with others." The strong emphasis on gains made in the interpersonal dimension might be interpreted as indicating that the group itself possibly functioned as an in vivo learning environment, where detainees learnt through sharing and discussion that they can be of support to one another.

Comparing the benefits highlighted by client feedback with the preset programme aims appears to indicate that the psychological support groups managed to satisfactorily fulfil two of the intended objectives (i.e. those relating to psychoeducation and imparting coping strategies). On the other hand, the data available is less clear about the programme's third aim. However, though the phenomenological aspect was not directly mentioned in the feedback, throughout the sessions a sizeable proportion of attendees did discuss their present experiences openly on a regular basis and showed appreciation for the opportunity to do so. In this regard, the group sessions served as a medium for detainees to express their voice on both an individual and collective level and their discourse painted a lucid picture of the lived experience of closed detention and its psychological sequelae. Group discussions about this subject helped participants and facilitators arrive at a shared understanding of this reality that then served as a guiding framework for the cognitive behavioural work conducted in later sessions. For example, discussions and exercises about cognitive distortions and emotional expression were focused on the issues and challenges that emerged from the open-ended exploration of participants' experiences. This component can hence be understood as setting the stage for the psychoeducative and cognitive behavioural work to be more targeted, and this could be one of the principal reasons behind the apparent benefits of the service in question.

Furthermore, observation of group processes provided insight into other ways the programme might have been of salutary benefit. As mentioned previously, the group sessions gave detainees the space to speak about themselves and how they see themselves in detention. In the initial sessions, they spoke mainly about the challenges of living in closed detention and explicated on the themes summarised above. Subsequently, later sessions served as a medium for these individuals to explore their psychological strengths and instances of coping.

Through group discussion about these issues, detainees offered encouragement and concrete advice to one another. Thus, the sessions' focus shifted from understanding and validating the lived experience of detention and detainees' psychological difficulties, to highlighting their strengths and potential for overcoming adversity on both an individual and collective level. Interestingly, another process appeared to occur in parallel; whilst attendees initially struggled with reflecting on their mental life and verbalising any acquired insight, as the sessions progressed they started displaying higher levels of psychological mindedness. These processes seem to serve as plausible explanations for why participants generally spoke about a greater confidence in their ability to cope at the end of the programme. Hence, it can be hypothesised that the sessions were of salutary benefit by providing detainees with an arena for negotiating a sense of self that is resilient to the experience of mandatory detention.

In terms of the specific psychological difficulties outlined beforehand, detainees verbally reported a reduction in the frequency and intensity of certain symptoms especially mood problems, insomnia, lethargy and the physical impact of anxiety. Based on the above analysis, it can be hypothesised that this effect is potentially linked to participants developing a greater understanding of the causes and modifiers of these difficulties and acquiring a set of pertinent skills and strategies applicable in detention. Furthermore, acquiring confidence in their psychological strengths and capacity to cope also seemed to play a crucial part by rendering the individual more prone to actively attempt to exert some influence over his/her own mental health. Finally, it is important to note that these sessions also had a salutary benefit by rendering detainees more prone to seek professional mental health support. Imparting an understanding of available mental health services and how these can be accessed and quelling notions of shame in seeking psychiatric/psychological treatment helped bring about this change.

\section{Psychological support groups as an intervention tool: challenges}

Providing this service in a Maltese detention context was particularly challenging in various respects. A prime challenge was certainly the unavailability of an adequate 
setting. As outlined beforehand, the majority of sessions took place in the centres' recreational areas, as the use of a private room external to detainees' living quarters was rarely possible. This meant that the psychological support groups generally took place in an environment characterised by restricted space, high noise levels and lack of privacy that made focusing on the session and sharing personal experiences more arduous for participants. Whilst this was a factor beyond the service provider's control that hindered the delivery of the programme, one might also argue that the fact that session environment was congruent with detention life had its advantages. This congruence offered facilitators the opportunity to directly witness detainees' living conditions and, albeit to a minimal degree, share their experience of detention life and may have rendered the groups more suited to function as an in vivo learning environment.

Another particular challenge was the initial diffidence of participants, probably stemming from their being unsure of the facilitators' intentions and the nature and ultimate aim of the programme. This diffidence must be understood in the context of a detainee's life whereby $\mathrm{s} /$ he may receive numerous visitors from both state and non-state agencies and due to language barriers is often confused about the purpose of these various visits. Overcoming this diffidence required the adoption of a targeted approach in the initial sessions, i.e. re-explaining the programme's aims and potential benefits, adopting a slower pace and allowing time for participants' queries. Although drop-outs inevitably occurred in the first three sessions, attendees' engagement improved with time as they acquired a greater understanding of the programme's scope.

The need for interpretation in certain groups presented another series of challenges, including the danger of certain nuances in participants' utterances being lost in translation and a slower overall session pace. Ensuring session effectiveness despite these difficulties required close collaboration between the facilitators and cultural mediators. In this regard, adopting the following procedures contributed to the smoother running of sessions: facilitators and cultural mediators discussing session content beforehand so as to aid the process of interpretation and holding a post session debriefing aimed at identifying areas for improvement.

Finally, differences between Western and sub-Saharan African cultures rendered the delivery of the psychological support groups even more complex. Relevant intercultural differences in this context pertained to individuals from sub-Saharan African cultures being less exposed to psychological constructs and having diverse conceptualisation of mental health. In this regard, the role of the cultural mediator extended beyond that of mere interpretation to serving as a cross-cultural bridge between facilitator and participant. Through this role, they enhanced the facilitator's comprehension of participants' opinions and disclosure by explaining culture-bound metaphors, idioms of distress, expressions and modes of behaving and facilitated the transmission of psychoeducative concepts by providing explanations that were grounded in an emic perspective.

\section{Addressing the psychological needs of detained migrants} From a mental health perspective, the forced detention of asylum seekers will, in all probability, always tend to have a worrying adverse psychological impact on an already vulnerable population. In this respect, mental health professionals have an important role to play in advocating for humane asylum procedures that are not detrimental to the mental health of the individuals concerned. In the interim, until the practice of administrative detention persists, the following suggestions for addressing detained migrants' psychological needs can be fruitful in ameliorating the care they receive.

The benefits highlighted by the evaluation of this programme's impact appear to encourage the use of similar interventions in detention centres. However, if the resources necessary to provide such a service are not available, the provision of psychoeducation sessions and selfhelp material can constitute fruitful alternative interventions. The former can serve to clarify misconceptions about psychological difficulties and address mental health stigma, thus helping to address one of the main barriers to accessing treatment. The latter can be of benefit by providing the detainee with a tool that enables them to take care of their own mental health. Although a self-help booklet can never substitute the need for psychotherapy or psychiatric treatment, the provision of self-help material that is simplified and relevant to the detention context can be an effective means for offering basic psychological support to the general detention population.

On a general note, the use of trained cultural mediators is certainly a fundamental prerequisite for the provision of effective mental health care to detained migrants. As discussed previously, in many cases, a significant divergence is present between the cultural conceptions of psychological health and distress prevalent in the migrant's culture of origin and those dominant in his/her host country. The majority of detained migrants would have been confined to a detention centre since their arrival in a new and alien cultural context and would hence be in great need of a cultural go-between, especially when seeking help for psychological difficulties. 
There is a high probability that not all mental health professionals in the host country will possess the background knowledge necessary to offer an effective service to detained migrants. In this respect, programmes aimed at increasing service providers' knowledge about the stressors associating with seeking asylum, the psychological impact of detention, the need to utilise cultural mediators and possible barriers to self-disclosure can help render these professionals better equipped to meet the needs of this unique client group.

The implementation of interventions that capitalise on an individual's personal strengths and capacities for coping may be highly effective for migrant populations that, despite the difficult situations they are exposed to, often possess adaptive skills and strategies that have spurred them on thus far. Since this approach is based on the search for personal capacities, it requires less didactic instruction and due to its ability to instil self-belief and empower may instigate a mode of coping that responds effectively to the harsh and taxing environment of forced detention.

\section{Limitations}

Whilst the overall feedback gathered was overwhelmingly positive, the data collected needs to be interpreted in light of the limitations of the feedback system used.

Unfortunately, the results described above pertain to only a proportion of those who attended the sessions as many attendees were released during the programme's duration and therefore did not fill the feedback form. ${ }^{9}$ Furthermore, there were a number of drop-outs throughout the programme, with these occurring mainly during the first three sessions, arguably during the stage where detainees were still in the process of ascertaining the nature of the programme. One should also take into consideration the fact that, as outlined above, a significant proportion of the detention population did not attend the psychological support groups from the outset. This seems to indicate that a filtering process occurred leaving only a proportion of those initially offered the opportunity attending the full programme.

It can therefore be hypothesised that the mean for how helpful participants found sessions was high, because the individuals questioned would have followed the programme till the end precisely since they considered it beneficial. Based on this reasoning, the best conclusion to draw from these results is that the programme seemed to be a useful tool for providing psychological support for detainees who chose to attend it and that this conclusion is not necessarily generalisable to the whole detention population.

A number of possible reasons will be offered for non-attendance or drop-out to give a wider context within which to evaluate the utility of the psychological support groups:

- The strong stigma about mental health problems prevalent in many sub-Saharan African cultures rendering the individual reluctant to even discuss these issues. A study by Slobodin et al. (2018) clearly indicated that fear of negative stigma was one of the reasons why mental health problems and the use of mental health services were silenced among asylum seekers.

- The strong apathy that sets in within the individual when $\mathrm{s} /$ he has been confined to such a restrictive environment.

- The relatively low level of education of many detainees meaning that they may be less accustomed to discussing in organised group formats.

It is also relevant to note that given the evaluative method chosen, no conclusions could be drawn about the efficacy of this intervention as a mode treatment for detainees with mental health problems. As such participants' feedback should be considered preliminary evidence about the potential usefulness of this group intervention as a means of support for asylum seekers during a period of administrative detention. Furthermore, the themes elicited can serve as a basis for the further development and refinement of what can become a valuable tool for working with this particular client group in this particular context.

\section{Conclusion}

For the forced migrant, the Maltese system of administrative detention means that, after having to deal with multiple assaults to their psychological health and integrity, they have to face yet another challenge. This challenge consists of a condition of limbo that, as recounted by the programme's participants, is experienced as inhuman, impersonal and degrading, as well as many times antithetical to their previous self-concept. Hence, detained migrants find themselves in dire need of psychological support, but from the service providers' perspective providing the necessary support is fraught with challenges and complexities, both pragmatic and technical. For psychological interventions to be effective in this context, they need to take into account the specific psychological needs of detained migrants, possible cultural and linguistic differences, the presence of pragmatic barriers and the phenomenological experience of detention. In this context, mental health practitioners find themselves navigating treacherous waters and may struggle about how to proceed. At such a juncture, validating the detainee's voice, acknowledging their humanity, personhood and strengths and striving to empower them will most certainly represent a fruitful avenue to pursue. 


\section{Endnotes}

${ }^{1}$ Definitions of the terms asylum seeker and refuge abound and to some extent vary according to a country's legal system. In the eyes of the Maltese legal system upon application for asylum in Malta, a migrant becomes an "asylum seeker" and would subsequently become a "refugee" if s/he is granted refugee status following their status determination procedure. When referring to the Maltese context, this paper will use the terms "migrant", "asylum seekers" and "refugees" in this manner, while when quoting other literature this paper will remain faithful to the original term used.

${ }^{2}$ In 2012, 1869 asylum seekers arrived by boat in comparison to 279 who were non-boat arrivals (Office of the Refugee Commissioner, Malta, 2013).

${ }^{3}$ Statistics based on the 14,774 arrivals between 2002 and 2011. In 2012, the majority of the 1869 arrivals were Eritrean and Somali (Office of the Refugee Commissioner, Malta, 2013).

${ }^{4}$ Twenty percent of total arrivals in 2011 and 2012 were women and children (Jesuit Refugee Service Malta 2013).

${ }^{5}$ Refugee Status is granted to those who according to the Geneva Convention (1951) are outside their country of nationality due to a well-founded fear of being persecuted because of reasons such as race, religion, war and violence and are unable to or, owing to such a fear, unwilling to avail themselves of the protection of that country.

${ }^{6}$ Subsidiary Protection is a form of international protection granted to asylum seekers in Malta who would face serious harm if returned to their country. Serious harm is defined "as the death penalty or execution; torture or cruel, inhuman and degrading treatment or punishment; or a serious and individual threat to a civilian's life because of indiscriminate violence in situations of external or internal armed conflict" (Jesuit Refugee Service Malta 2008, p.6).

${ }^{7}$ The role of the cultural mediator in this case was to provide interpretation in the asylum seeker's tongue as well as provide information about participants' cultural background that can aid the psychologist's understanding of the participants' input during the sessions. These cultural mediators were trained by JRS, Malta, but had no formal accreditation.

${ }^{8}$ Ad verbatim excerpts from participant feedback forms.

${ }^{9}$ These attendees were mainly released after being afforded protection or being recognised as vulnerable individuals.

\section{Abbreviations}

CBT: Cognitive behaviour therapy; JRS: Jesuit Refugee Service; PTSD: Posttraumatic stress disorder; QoL: Quality of life; THP: Temporary Humanitarian Protection
Acknowledgements

Not applicable.

Funding

No funding sources were obtained.

Availability of data and materials

Dataset can be found on https://figshare.com/s/fc895f087a07f0b5704a

\section{Authors' contributions}

JC conceived of the study, reviewed relevant literature, analysed the data collected, compiled the results and drafted the manuscript. AR conceived of the study, reviewed relevant literature, compiled the results and drafted the manuscript. Both authors read and approved the final manuscript.

\section{Authors' information}

J.C - warranted Counselling Psychologist, employed by JRS Malta and working as a senior visiting lecturer at University of Malta with the Department of Psychology.

A.R - warranted Counselling Psychologist, employed by JRS Malta and working as a senior visiting lecturer at University of Malta with the Department of Psychology.

\section{Competing interests}

The authors declare that they have no competing interests.

\section{Publisher's Note}

Springer Nature remains neutral with regard to jurisdictional claims in published maps and institutional affiliations.

Received: 3 April 2018 Accepted: 24 January 2019

Published online: 19 February 2019

\section{References}

Alcock M (2003) Refugee trauma: the assault on meaning. Psychodyn Pract 9: 291-306

Amnesty International (2013) Amnesty International report 2013. In: The state of the world's human rights-Malta. Available via Google Scholar. http://files. amnesty.org/air13/AmnestyInternational_AnnualReport2013_complete_en. pdf. Accessed 5 June 2013

Bogic M, Njoku A, Priebe S (2015) Long-term mental health of war-refugees: a systematic literature review. BMC International Health and Human Rights, in press.

Castro FG, Murray KE (2010) Cultural adaptation and resilience: controversies, issues, and emerging models. In: Reich JW, Zautra AJ, Hall JS (eds) Handbook of adult resilience. The Guilford Press, USA

Curling P (2005) The effectiveness of empowerment workshops with torture survivors. Torture 15:9Y15

Droždek B, Kamperman AM, Bolwerk N, Tol WA, Kleber RJ (2012) Group therapy with male asylum seekers and refugees with posttraumatic stress disorder: a controlled comparison cohort study of three day-treatment programs. J Nerv Ment Dis 200:758-765

Farias, P (1991) Emotional distress and its socio-political correlates in Salvadoran refugees: analysis of a clinical sample. Culture, Medicine and Psychiatry 15: 167-192.

Fazel M, Silove D (2006) Detention of refugees. Br Med J 332:251-252

Fazel M, Wheeler J, Danesh J (2005) Prevalence of serious mental disorder in 7000 refugees resettled in western countries: a systematic review. Lancet 365:1309-1314.

Fischman Y (2008) Secondary trauma in the legal professions: a clinical perspective. Torture 18:107-115

Goffman E (1961) Asylums: essays on the social situation of mental patients and other inmates. Anchor Books, New York

Human Rights Watch (2012) Malta: migrant detention violates rights. Available via Google Scholar.http://www.hrw.org/news/2012/07/18/malta-migrantdetention-violates-rights. Accessed 10 Feb 2014

Huntley AL, Araya R, Salisbury C (2012) Group psychological therapies for depression in the community: systematic review and meta-analysis. $\mathrm{Br} \mathrm{J}$ Psychiatry 200:184-190

Hussain D, Bhushan B (2009) Development and validation of the refugee trauma experience inventory. Psychol Trauma Theory Res Pract Policy 1:107-117 
Jesuit Refugee Service Europe (2010a) Becoming vulnerable in detention. JRS European Regional Office Press, Brussels

Jesuit Refugee Service Europe (2010b) Becoming vulnerable in detention: National report Malta. JRS European Regional Office Press, Brussels

Jesuit Refugee Service Malta (2008) Asylum in Malta: what you should know. Think Design Limited, Malta

Jesuit Refugee Service Malta (2013) Improving care and protection for female migrants and asylum seekers arriving in Malta. JRS Office, Malta

Jesuit Refugee Service Malta (2014) Detention in Malta. Available via Google Scholar. http://www.jrsmalta.org/content.aspx?id=225267. Accessed 12 Jan 2014

Keller AS, Rosenfeld B, Trinh-Shvrin C, Meserve C, Sachs E, Leviss J et al (2003) Mental health of detained asylum seekers. Lancet 362:1721-1723

Laban CJ, Komproe IH, Gernaat HBPE, de Jong JTVM (2008) Impact of a long asylum procedure on quality of life, disability and physical health in Iraqi asylum seekers in the Netherlands. Soc Psychiatry Psychiatr Epidemiol. 43: 507-515.

Lim RF, Koike AK (2010) Psychotherapy and refugees. In: Bhugra D, Craig T, Bhui $K$ (eds) Mental health of refugees and asylum seekers. Oxford University Press, Oxford

Main N, Elliot S, Brown J (2005) Comparison of three different approaches used in large-scale stress workshops for the general public. Behav Cogn Psychother 33:299-310

Manneschmidt S, Griese K (2009) Evaluating psychosocial group counselling with Afghan women: is this a useful intervention? Torture 19:41-50

Muecke MA (1992) New paradigms for refugee health problems. Soc Sci Med 35: 515-523

Office of the Refugee Commissioner, Malta (2013) Studying integration efforts of beneficiaries of international protection and identifying areas where special input is needed. Paper presented at the ERF co-funded project conference putting integration into perspective, Valletta, Malta, 19 June 2013

Otto MW, Hinton DE, Korbly NB, Chea A, Ba P, Gershuny BS et al (2003) Treatment of pharmacotherapy-refractory posttraumatic stress disorder among Cambodian refugees: a pilot study of combination treatment with cognitive-behaviour therapy vs. sertraline alone. Behav Res Ther 41:12711276

Porter M, Haslam N (2005) Predisplacement and postdisplacement factors associated with mental health of refugees and internally displaced persons: a meta-analysis. J Am Med Assoc 294:602-612

Pourgourides CK, Sashidharan SP, Bracken PJ (1996) A second exile: the mental health implications of detaining asylum seekers in the United Kingdom. North Birmingham NHS Trust, Birmingham

Robjant K, Hassan R, Katona C (2009) Mental health implications of detaining asylum seekers: systematic review. Br J Psychiatry 194:306-321

Salinsky M, Dell S (2001) Protection not prison: torture survivors detained in the UK. Medical Foundation for the Care of Victims of Torture, London

Silove D, Steel Z, Watters C (2000) Policies of deterrence and the mental health of asylum seekers. J Am Med Assoc 284:604-611

Slobodin O, Ghane S, De Jong JT (2018) Developing a culturally sensitive mental health intervention for asylum seekers in the Netherlands: a pilot study. Intervention 16:86-94

Steel Z, Chey T, Silove D, Marnane C, Bryant R, van Ommeren M (2009) Association of torture and other potentially traumatic events with mental health outcomes among populations exposed to mass conflict and displacement: a systematic review and meta-analysis. J Am Med Assoc 302: $537-549$

Watters C (2007) The mental health care of asylum seekers and refugees. In: Knapp M, McDaid D, Mossialos E, Thornicroft G (eds) Mental health policy and practice across Europe. McGraw-Hill Open University Press, Berkshire

Weine S, Kulauzovic Y, Klebic A, Besic S, Mujagic A, Muzurovic J, Rolland J et al (2008) Evaluating a multiple-family group access intervention for refugees with PTSD. J Marital Fam Ther 34:149-164

Weine S, Muzurovic N, Kulauzovic Y, Besic S, Lezic A, Mujagic A, Knafl K et al (2004) Family consequences of refugee trauma. Fam Process 43:147-160

White J, Keenan M, Brooks N (1992) Stress control: a controlled comparative investigation of large group therapy for generalized anxiety disorder. Behav Cogn Psychother 20:97-114

\section{Submit your manuscript to a SpringerOpen ${ }^{\circ}$ journal and benefit from:}

- Convenient online submission

- Rigorous peer review

- Open access: articles freely available online

- High visibility within the field

- Retaining the copyright to your article

Submit your next manuscript at $\boldsymbol{\nabla}$ springeropen.com 